\title{
A universal chromosome identification system for maize and wild Zea species
}

Guilherme T. Braz ${ }^{1}$, Lívia do Vale Martins ${ }^{1}$, Tao Zhang $^{2}$, Patrice S. Albert ${ }^{3}$, James A. Birchler ${ }^{3}$, and Jiming Jiang $1,4,5, *$

${ }^{1}$ Department of Plant Biology, Michigan State University, East Lansing, MI 48824, USA

2 Jiangsu Key Laboratory of Crop Genomics and Molecular Breeding/Key Laboratory of Plant Functional Genomics of Ministry of Education, Yangzhou University, 225009, China

3 Division of Biological Sciences, University of Missouri, Columbia, MO 65211, USA

4 Department of Horticulture, Michigan State University, East Lansing, MI 48824, USA

5 Michigan State University AgBioResearch, East Lansing, MI 48824, USA

*Address correspondence to: jiangjm@msu.edu 


\begin{abstract}
Maize was one of the first eukaryotic species in which individual chromosomes can be identified cytologically, which made maize one of the oldest models for genetics and cytogenetics research. Nevertheless, consistent identification of all 10 chromosomes from different maize lines as well as from wild Zea species remains a challenge. We developed a new technique for maize chromosome identification based on fluorescence in situ hybridization (FISH). We developed two oligonucleotide-based probes that hybridize to 24 chromosomal regions. Individual maize chromosomes show distinct FISH signal patterns, which allow universal identification of all chromosomes from different Zea species. We developed karyotypes from three Zea mays subspecies and two additional wild Zea species based on individually identified chromosomes. A paracentric inversion was discovered on the long arm of chromosome 4 in Z. nicaraguensis and Z. luxurians based on modifications of the FISH signal patterns. Chromosomes from these two species also showed distinct distribution patterns of terminal knobs compared to other Zea species. These results support that Z. nicaraguensis and $Z$. luxurians are closely related species.
\end{abstract}

Keywords: FISH, oligo-FISH, chromosome identification, karyotype, maize 


\section{Introduction}

A reliable and efficient system for chromosome identification is the foundation for cytogenetic research. Maize (Zea mays) and common fruit fly (Drosophila melanogaster) became the most important model species for genetic and cytogenetic research in early $20^{\text {th }}$ century because individual chromosomes could be readily identified in both species (Bridges 1935; McClintock 1929). However, the identification of individual chromosomes relied on meiotic pachytene chromosomes in maize and salivary polytene chromosomes in Drosophila. Thus, the chromosome identification methodologies developed in these two model species were not applicable in most of the other eukaryotes. Preparation and identification of pachytene chromosomes is technically demanding, thus, cytogenetic research in most plant species relies on mitotic metaphase chromosomes. Most of the chromosome identification systems based on mitotic metaphase chromosomes were developed after 1970s using either chromosome banding or DNA in situ hybridization techniques (Jiang and Gill 1994).

Although maize chromosomes can be individually identified based on their morphology (McClintock 1929), there are ambiguities. Chromosome banding techniques were developed in 1980s and 1990s for maize chromosome identification (de Carvalho and Saraiva 1993, 1997; Deaguiarperecin and Vosa 1985; Jewell and Islam-Faridi 1994; Kakeda et al. 1990; Rayburn et al. 1985; Ward 1980). C-banding, the most popular banding technique in plants, revealed mostly the knob-related regions on maize chromosomes (Deaguiarperecin and Vosa 1985; Jewell and Islam-Faridi 1994; Rayburn et al. 1985). Maize chromosomes showed a limited number of Cbands that are also highly polymorphic among different maize varieties. Therefore, a standard banding pattern cannot be established for routine maize chromosome identification.

Fluorescence in situ hybridization (FISH) gradually replaced chromosome banding to become the most popular technique for chromosome identification in plants (Jiang and Gill 2006). Repetitive DNA sequences and large-insert DNA clones, such as bacterial artificial chromosome (BAC) clones, have been most commonly used as FISH probes in maize (Amarillo and Bass 2007; Chen et al. 2000; Figueroa and Bass 2012; Koumbaris and Bass 2003; Lamb et al. 2007b; Sadder and Weber 2001; Wang et al. 2006). Kato et al. (2004) used a combination of several repetitive DNA sequences as FISH probes to identify all 10 maize chromosomes in the same metaphase cells. This repeat-based FISH method was successfully used to identify chromosomes in various maize lines as well as wild Zea species and subspecies (Albert et al. 
2010; Kato et al. 2004). The FISH signal patterns of some repeats, however, are highly polymorphic among different maize lines, which may cause difficulties or errors in chromosome identification.

Recently, a new class of FISH probes was developed based on pooled single-copy oligonucleotides (oligo) (Jiang 2019). If the genomic sequence is available from a target species, oligos, typically 40-50 bp long, specific to a chromosomal region or to an entire chromosome can be synthesized in parallel and labeled as a FISH probe (Han et al. 2015). Oligo-FISH probes have been successfully developed in several plant species for various types of cytogenetic studies (Albert et al. 2019; Bi et al. 2020; He et al. 2018; Hou et al. 2018; Martins et al. 2019; Meng et al. 2018; Qu et al. 2017; Simonikova et al. 2019; Song et al. 2020; Xin et al. 2018; Xin et al. 2020). A FISH probe can include oligos derived from multiple regions from multiple chromosomes (Braz et al. 2018). Such oligo-FISH probes generate specific hybridization patterns on individual chromosomes, which resemble distinct barcodes on different chromosomes. This chromosome identification strategy has been successfully demonstrated in potato (Braz et al. 2018), rice (Liu et al. 2020) and sugarcane (Meng et al. 2020). Here, we report the development of similar barcode FISH probe in maize. We demonstrate that barcode FISH can be used as an universal chromosome identification system for maize and all wild Zea species. The FISH signal pattern is not affected by the DNA polymorphisms existing among different maize varieties or even among different species. Using this system we were able to establish karyotypes of several different Zea species and subspecies and to discover a paracentric inversion specific to two wild Zea species.

\section{Materials and Methods}

\section{Plant Materials}

We included the following Zea species and subspecies in chromosome identification and karyotyping studies: Z. mays (inbred B73), Z. mays ssp. parviglumis (Ames 21826), Z. mays ssp. mexicana (Ames 21851), Z. mays ssp. huehuetenangensis (PI 441934), Z. diploperennis (PI 462368), Z. nicaraguensis (PI 615697), and Z. luxurians (PI 422162). Additional species included in the study were the tetraploid species Z. perennis (Ames 21874), Tripsacum dactyloides (PI 421612), and Sorghum bicolor (PI 564163). Seeds of all wild species were originally from the Germplasm Resources Information Network (GRIN) and the National Genetics Resource Program (Ames, Iowa). 


\section{Oligo-FISH probe design}

The two oligo-based barcode probes were designed following our published procedure (Albert et al. 2019) using Chorus software (https://github.com/forrestzhang/Chorus). Briefly, the maize genome sequence was divided into 45-nt long oligos in step size of $3 \mathrm{nt}$. The short sequence reads were mapped back to the genome using BWA (Burrows-Wheeler Alignment tool) (Li and Durbin 2009). Oligos mapped to two or more locations (with 70\% homology) were eliminated. All repetitive sequences related oligos were filtered out from the oligo set using $k$ mer method (Albert et al. 2019). We selected oligos from 24 chromosomal regions to create a barcode for all 10 maize chromosomes (Figure 1a). Each selected region contains approximately 2000 oligos. We selected the chromosomal regions that are relatively enriched with single copy sequences. Each of the 24 selected regions spans 1.3-3.3 Mb (Table S1). If two regions are designed from the same chromosomal arm, we then intended to separate the two regions as far as possible. The two regions on eight different chromosomal arms (Figure 1a) are separated 28.266.4 Mb (Table S1).

\section{Oligo-FISH}

Root tips harvested from plants grown in greenhouse were treated with nitrous oxide at a pressure of $160 \mathrm{psi}(\sim 10.9 \mathrm{~atm})$ for $2 \mathrm{hrs}$. and $20 \mathrm{~min}$ (Kato 1999), fixed in fixative solution (3 ethanol : 1 acetic acid) and kept at $-20^{\circ} \mathrm{C}$. Root tips were digested using an enzymatic solution composed by 4\% cellulase (Yakult Pharmaceutical, Japan), 2\% pectinase (Sigma-Aldrich Co., USA) and $2 \%$ pectolyase (Plant Media, USA) for two hours at $37^{\circ} \mathrm{C}$ and slides were prepared using the stirring method (Ross et al. 1996).

Two oligo-based FISH probes were labeled according to published protocols (Han et al. 2015) and hybridized to metaphase chromosomes (Cheng et al. 2002). Biotin- and digoxygeninlabeled probes were detected by anti-biotin fluorescein (Vector Laboratories, Burlingame, California) and anti-digoxygenin rhodamine (Roche Diagnostics, Indianapolis, Indiana), respectively. Chromosomes were counterstained with 4,6-Diamidino-2-phenylindole (DAPI) in VectaShield antifade solution (Vector Laboratories). FISH images were captured using a QImaging Retiga EXi Fast 1394 CCD camera attached to an Olympus BX51 epifluorescence 
microscope. Images were processed with Meta Imaging Series 7.5 software. The final contrast of the images was processed using Adobe Photoshop software.

\section{Karyotyping}

We used 7-10 complete metaphase cells of Z. mays and its relatives (Z. mays ssp. parviglumis, Z. mays ssp. mexicana, Z. mays ssp. huehuetenangensis, Z. nicaraguensis, $Z$. luxurians) to measure the short (S) and long (L) arms of individual chromosomes using DRAWID software version 0.26 (Kirov et al. 2017). The measurements were used to determine the total length of each chromosome $(t l=S+L)$, total length of entire set of chromosomes $(T L=$ $\left.\sum t l\right)$, arm ratio $(A R=L / S)$ of each chromosome, and relative length of each chromosome $(R L=$ $t l / T L \times 100)$. Chromosomal knobs were identified as DAPI-positive bands. Chromosomes were classified based on arm ratio following Levan et al. (1964).

\section{Results}

\section{Development of oligo-FISH probes for maize chromosome identification}

We developed two oligo-based FISH probes to facilitate simultaneous identification of all 10 maize chromosomes in the same metaphase cells. These two probes contained a total of 50,082 oligos (45 nt) designed from single copy sequences in the maize genome (Schnable et al. 2009). The oligos were selected from 24 chromosomal regions (Figure 1a), each containing 1978-2282 oligos and spanning 1.3-3.3 megabase (Mb) of DNA sequences (Table S1). The oligos were synthesized as two separate pools, containing 25,059 (red probe) and 25,023 (green probe) oligos, respectively. FISH using these two probes generated 24 distinct signals on metaphase chromosomes prepared from maize inbred B73 (Figure 1b). The chromosomal positions of the FISH signals matched with predicted positions on the 10 pseudomolecules, which allowed us to readily distinguish 10 individual chromosomes in the same metaphase cell (Figure 1c). Several chromosomal arms contained two signals, which were well separated on metaphase chromosomes. The two signals designed on the short arm of chromosome 4 were separated by $28.2 \mathrm{Mb}$, representing the shortest distance among the paired signals (Table S1). These two signals appeared to be fused only on some highly condensed metaphase chromosomes.

\section{Chromosome identification in different Zea species}


The two oligo-FISH probes were used to identify chromosomes from several Z. mays subspecies and wild Zea species known as “teosintes”, including Z. mays ssp. parviglumis (Figure 2a), Z. mays ssp. mexicana (Figure 2b), Z. mays ssp. huehuetenangensis (Figure 2c), $Z$. diploperennis (Figure 2d), Z. nicaraguensis (Figure 2e), and Z. luxurians (Figure 2f). We observed a nearly identical FISH signal pattern on chromosomes from all Zea species and subspecies (Figure 3). We also performed oligo-FISH analysis in the tetraploid species $Z$. perennis $(2 \mathrm{n}=4 \mathrm{x}=40)$. Similarly, the conserved FISH signal pattern was observed on all 10 sets of homologous chromosomes (Figure 4). Thus, these two probes allowed us to identify all chromosomes from all Zea taxa.

\section{A putative inversion detected on long arm of chromosome 4}

We detected some noticeable chromosome level differences based on the signal patterns of oligo-FISH. One distinct FISH signal pattern change was observed on the long arm of chromosome 4 from Z. nicaraguensis and Z. luxurians (Figure 3). One green signal was designed from the distal end on the long arm of chromosome 4 (at 234-236 Mb of chromosome 4, which is $242 \mathrm{Mb}$ long). This signal was detected at the end of the long arm of maize chromosome 4 (Figure 1c). However, chromosome 4 of Z. nicaraguensis and Z. luxurians did not contain this terminal signal. It instead contains two interstitial green signals on the long arm (Figure 3). Thus, a paracentric inversion may have occurred in the long arm, which would relocate the terminal signal into an interstitial position.

\section{Chromosomal difference revealed by oligo-FISH}

The number, size, and distribution of metaphase-visible "knobs" were the most prominent factor resulting in the difference observed on the same chromosome from different species (Figure 3). Although the polymorphism of knob distribution among different maize lines is well known (Adawy et al. 2004; Albert et al. 2010), distinct knob distribution patterns were observed in some species. For example, a knob was detected between the two red signals on the long arm of chromosome 5 in Z. mays ssp. parviglumis. By contrast, a knob was observed at the distal end of the long arm of chromosome 5 in Z. nicaraguensis and Z. luxurians (Figure 3, yellow arrowheads). A similar difference of FISH signal position among species, caused by the presence of a terminal vs. subterminal knob, was observed on the distal regions of several other 
chromosomal arms, including the short arm of chromosome 2, and long arm of chromosomes 1 , 2, 3, 6, 7, 8, and 9 (Figure 3). Interestingly, most of the terminal knobs in $Z$. nicaraguensis and $Z$. luxurians were located distal to the FISH signals, suggesting a close structural similarity of these two species (Figure 3).

In several cases, a major knob was observed only on one copy of a pair of homologous chromosomes. For example, the long arm of chromosome 3 contained two red signals (Figure 1a). The two red signals were separated by a major knob in one copy of chromosome 3 in $Z$. mays ssp. parviglumis. This knob was not visible in the second copy of chromosome 3 (Figure 3). The distance between the two red signals appeared to be significantly different on the two chromosomes due to the presence of this heterozygous knob. A similar heterozygous knob was observed on several other chromosomes among different species (Figure 3, red arrowheads).

The size of a knob can also be visibly different on two homologous chromosomes. For example, a terminal knob was observed on the long arm of chromosome 10 in several species. The size of this knob appeared to be distinctly different on the two copies of chromosome 10 in $Z$. nicaraguensis (Figure 3, white arrowheads). Both presence/absence and size polymorphism of major knobs can result in a significant difference of the size of the homologous chromosomes.

\section{The karyotypes of different Zea species and subspecies}

Unambiguous identification of all chromosomes in the same metaphase cells allowed us to develop karyotypes based on individually identified chromosomes from all Zea species and subspecies (Tables 1, 2). Each karyotype was developed based on measurements of all chromosomes in 7-10 complete metaphase cells. Not surprisingly, all Zea species and subspecies shared a highly similar karyotype with similar relative length and arm ratio of all 10 chromosomes (Tables 1, 2). Chromosomes 1, 4, 5, and 10 are morphologicaly conserved, and are metacentric (Chr. 1, 4, and 5) and submetacentric (Chr. 10) in all species (Table 2). The other chromosomes are also similar but with minor variations among different species. Chromosomes 2 and 3 are metacentric in all species, except in Zea mays ssp. mexicana (submetacentric). Chromosome 7 is submetacentric in all species, except $Z$. mays ssp. mexicana (metacentric). Chromosome 8 is submetacentric in all the species, except in $Z$. mays ssp. huehuetenangensis (metacentric). Chromosome 9 is submetacentric in all the species, except $Z$. 
mays ssp. parviglumis and Z. mays ssp. huehuetenangensis (metacentric). Chromosome 6 is the only satellite chromosomes in all species.

\section{Application of the oligo probes in distantly related species}

We explored the potential of the barcode probes for chromosome identification in species that are more distantly related to maize. We first tested the two maize probes in hybridization to metaphase chromosomes prepared from Tripsacum dactyloides $(2 \mathrm{n}=2 \mathrm{x}=36)$, a species from a sister genus related to Zea and diverged from maize about 4.5 million years (MYs) (Hilton and Gaut 1998). The two oligo-FISH probes produced punctuated signals on most of chromosomes, which allowed to identify few putative homoeologous chromosomes (Figure 5a). However, most of the signals were not as strong as those on maize chromosomes. In addition, strong backgorund signals were observed on most chromosomes. Several T. dactyloides chromosomes lacked unambiguous signals (Figure 5a). Thus, the maize oligo probes were not useful to identify individual T. dactyloides chromosomes.

Sorghum (Sorghum bicolor) and maize diverged from maize ancestors for about 12 MYs (Swigonova et al. 2004). The two maize probes produced background signals on all sorghum chromosomes (Figure 5b). Only few of the 10 sorghum chromosomes generated consistent and distinct FISH signal patterns. Thus, the maize probes were not useful to identify individual sorghum chromosomes.

\section{Discussion}

FISH has become the most popular methodology for chromosome identification in plants (Jiang and Gill 2006). Different FISH methodologies have different advantages and drawbacks. BACs and repetitive DNA sequences are the two most common types of FISH probes used in chromosome identification. Unfortunately, BACs are not ideal probes for plant species with large genomes because of the presence of large percentages of repetitive DNA sequences in the clones (Janda et al. 2006; Zhang et al. 2004). Repeat-based chromosome identification systems have been established in several plant species (Findley et al. 2010; Fradkin et al. 2013; Kato et al. 2004; Lengerova et al. 2004; Li et al. 2014; Mukai et al. 1993; Xiong and Pires 2011). Repeatbased systems reveal polymorphisms for the constituent sequences but are not genotype independent. By contrast, single-gene karyotyping probes are genotype independent (Danilova 
and Birchler 2008; Danilova et al. 2014; Lamb et al. 2007a). Similarly, the FISH signal patterns derived from barcode probes are not polymorphic among different varieties in the same species. A modified pattern would indicate a potential chromosomal rearrangement associated with a cultivar. In addition, barcode probes can also be used for chromosome identification in genetically related species (Braz et al. 2018; Liu et al. 2020) (Figure 2). Most importantly, barcode FISH probes can be developed readily in any plant species with a sequenced genome.

We have recently demonstrated that barcode FISH probes developed based on potato sequences were useful to identify individual homoeologous chromosomes from distantly related species, including tomato (Solanum lycopersicum), which diverged from potato for 5-8 MYs (Sarkinen et al. 2013; Wang et al. 2008). The potato barcode probes also generated punctuate signals but with a visible background on chromosomes from eggplant (Solanum melongena), which diverged from potato for 15.5 MYs (Wu and Tanksley 2010). A total of 54672 oligos were used in the two potato barcode probes, including 16489 oligos (30\%) designed from coding sequences of potato genes. In contrast, among the 50,082 maize oligos, only 5525 oligos (11\%) are associated with coding sequences. This may explain the fact that the maize barcode probes generate strong background signals on chromosomes of $T$. dactyloides, which diverged from maize for only 4.5 MYs (Hilton and Gaut 1998). Therefore, the percentage of oligos designed from highly conserved DNA sequences is critical to extend the value of barcode FISH probes for chromosome identification in distantly related species.

One of the key factors for developing successful barcode probes is to restrict the oligos within a chromosomal region as narrow as possible. A bright FISH signal can be generated from 1000-2000 oligos. However, these oligos need to be distributed within a few Mb. Each of the 24 regions of our maize barcode span 1.3-3.3 Mb of DNA sequences (Table S1). It can be challenging to identify 1000-2000 unique oligos within repetitive chromosomal regions, especially in plant species with very large and complex genomes. Thus, it may become necessary to develop as many oligos as possible within the small islands of genic or single copy sequences in largely repetitive regions. For example, oligos, or overlapping oligos, can be designed from both strands of the DNA sequences within the single copy sequences.

It is not surprising that all Zea species and subspecies share a highly conserved karyotype (Tables 1, 2) since these species have diverged for only $~ 150,000$ years (Ross-Ibarra et al. 2009). Phylogenetic studies based on microsatellite markers indicated that Z. nicaraguensis and $Z$. 
luxurians are closely related species and Z. nicaraguensis could be treated as a subspecies of $Z$. luxurians (Fukunaga et al. 2005). This conclusion was supported by our barcode FISH and karyotyping data. Terminal knobs, which are distal to the terminal FISH signals, were identified on several chromosome arms, including the long arms of chromosomes 2, 5, 6, 7, 9, and 10 in both Z. nicaraguensis and Z. luxurians (see also Albert et al. 2010). Interestingly, these terminal knobs were not observed at the same chromosomal positions in other species/subspecies (Figure 3). In contrast, subterminal knobs, which are proximal to the terminal FISH signals, were observed on the long arms of chromosomes 2, 5, 6, 7, and 9 (Figure 3) in several species/subspecies, but not in Z. nicaraguensis and Z. luxurians. Thus, Z. nicaraguensis and Z. luxurians share a similar knob distribution pattern.

A putative paracentric inversion was detected in the long arm of chromosome 4 in both $Z$. nicaraguensis and Z. luxurians. Poggio (2005) developed hybrids between Z. luxurians and three subspecies of Z. mays (Z. mays ssp. parviglumis, Z. mays ssp. mexicana, Z. mays ssp. mays) and analyzed meiotic chromosome pairing in hybrids. Interestingly, the most frequent chromosome pairing configuration at metaphase I of meiosis in the hybrids was eight bivalents and four univalents (Poggio et al. 2005), suggesting major structural changes associated potentially with two Z. luxurians chromosomes. In addition, bridges and broken chromosome fragments were observed at anaphase I and II (Poggio et al. 2005), which are typical products associated with heterozygous paracentric inversions (Sybenga 1972). In addition, genetic linkage mapping data showed that the chromosome 4 from $Z$. nicaraguensis and Z. luxurians share the synteny but they contain a large inversion relative to maize chromosome 4 (Mano and Omori 2013). These results support a paracentric inversion associated with the long arm of chromosome 4. It is likely that additional Z. nicaraguensis/Z. luxurians chromosomes are involved in major structural changes compared to others in the Zea lineage. An inversion was found on the long arm of chromosome 3 based on pachytene chromosome analysis (Fang et al. 2012; Ting 1965), although chromosome 3 shares an identical barcode FISH pattern across all Zea species and subspecies (Figure 3). Indeed, because the barcode uses only a few well-spaced landmarks per chromosome, chromosomal aberrations, especially those restricted in regions proximal to the landmarks, will go undetected. Barcodes with more dense landmarks and in combination with whole chromosome paints (Albert et al. 2019) will be powerful tools to identify and characterize chromosomal rearrangements occurred during the evolution of Zea species. 


\section{Acknowledgements:}

This research is supported by NSF grant IOS-1444514 and MSU startup funds to J.J.

\section{References}

Adawy SSM, Stupar RM, Jiang J (2004) Fluorescence in situ hybridization of knob-associated DNA elements analysis reveals multiple loci in one-knob and knobless maize lines. $\mathrm{J}$ Histochem Cytochem 52:1113-1116

Albert PS, Gao Z, Danilova TV, Birchler JA (2010) Diversity of chromosomal karyotypes in maize and its relatives. Cytogenet Genome Res 129:6-16

Albert PS, Zhang T, Semrau K, Rouillard JM, Kao YH, Wang CJR, Danilova TV, Jiang JM, Birchler JA (2019) Whole-chromosome paints in maize reveal rearrangements, nuclear domains, and chromosomal relationships. P Natl Acad Sci USA 116:1679-1685

Amarillo FIE, Bass HW (2007) A transgenomic cytogenetic sorghum (Sorghum propinquum) bacterial artificial chromosome fluorescence in situ hybridization map of maize (Zea mays L.) pachytene chromosome 9, evidence for regions of genome hyperexpansion. Genetics 177:1509-1526

Bi YF, Zhao QZ, Yan WK, Li MX, Liu YX, Cheng CY, Zhang L, Yu XQ, Li J, Qian CT, Wu YF, Chen JF, Lou QF (2020) Flexible chromosome painting based on multiplex PCR of oligonucleotides and its application for comparative chromosome analyses in Cucumis. Plant J:10.1111/tpj.14600

Braz GT, He L, Zhao HN, Zhang T, Semrau K, Rouillard JM, Torres GA, Jiang JM (2018) Comparative Oligo-FISH mapping: an efficient and powerful methodology to reveal karyotypic and chromosomal evolution. Genetics 208:513-523

Bridges CB (1935) Salivary chromosome maps with a key to the banding of the chromosomes of Drosophila melanogaster. J Hered 26:60-64

Chen CC, Chen CM, Hsu FC, Wang CJ, Yang JT, Kao YY (2000) The pachytene chromosomes of maize as revealed by fluorescence in situ hybridization with repetitive DNA sequences. Theor Appl Genet 101:30-36

Cheng ZK, Buell CR, Wing RA, Jiang JM (2002) Resolution of fluorescence in-situ hybridization mapping on rice mitotic prometaphase chromosomes, meiotic pachytene chromosomes and extended DNA fibers. Chromosome Res 10:379-387

Danilova TV, Birchler JA (2008) Integrated cytogenetic map of mitotic metaphase chromosome 9 of maize: resolution, sensitivity, and banding paint development. Chromosoma 117:345-356

Danilova TV, Friebe B, Gill BS (2014) Development of a wheat single gene FISH map for analyzing homoeologous relationship and chromosomal rearrangements within the Triticeae. Theor Appl Genet 127:715-730

de Carvalho CR, Saraiva LS (1993) A new heterochromatin banding pattern revealed by modified HKG banding technique in maize chromosomes. Heredity 70:515-519

de Carvalho CR, Saraiva LS (1997) High-resolution HKG-banding in maize mitotic chromosomes. J Plant Res 110:417-420 
Deaguiarperecin MLR, Vosa CG (1985) C-banding in maize II. Identification of somatic chromosomes. Heredity 54:37-42

Fang Z, Pyhajarvi T, Weber AL, Dawe RK, Glaubitz JC, Gonzalez JDS, Ross-Ibarra C, Doebley J, Morrell PL, Ross-Ibarra J (2012) Megabase-scale inversion polymorphism in the wild ancestor of maize. Genetics 191:883-U426

Figueroa DM, Bass HW (2012) Development of pachytene FISH maps for six maize chromosomes and their integration with other maize maps for insights into genome structure variation. Chromosome Res 20:363-380

Findley SD, Cannon S, Varala K, Du JC, Ma JX, Hudson ME, Birchler JA, Stacey G (2010) A fluorescence in situ hybridization system for karyotyping soybean. Genetics 185:727-744

Fradkin M, Ferrari MR, Espert SM, Ferreira V, Grassi E, Greizerstein EJ, Poggio L (2013) Differentiation of triticale cultivars through FISH karyotyping of their rye chromosomes. Genome 56:267-272

Fukunaga K, Hill J, Vigouroux Y, Matsuoka Y, Sanchez J, Liu KJ, Buckler ES, Doebley J (2005) Genetic diversity and population structure of teosinte. Genetics 169:2241-2254

Han YH, Zhang T, Thammapichai P, Weng YQ, Jiang JM (2015) Chromosome-specific painting in cucumis species using bulked oligonucleotides. Genetics 200:771-779

He L, Braz GT, Torres GA, Jiang JM (2018) Chromosome painting in meiosis reveals pairing of specific chromosomes in polyploid Solanum species. Chromosoma 127:505-513

Hilton H, Gaut BS (1998) Speciation and domestication in maize and its wild relatives: Evidence from the globulin-1 gene. Genetics 150:863-872

Hou LL, Xu M, Zhang T, Xu ZH, Wang WY, Zhang JX, Yu MM, Ji W, Zhu CW, Gong ZY, Gu MH, Jiang JM, Yu HX (2018) Chromosome painting and its applications in cultivated and wild rice. Bmc Plant Biol 18:110

Janda J, Safar J, Kubalakova M, Bartos J, Kovarova P, Suchankova P, Pateyron S, Cihalikova J, Sourdille P, Simkova H, Faivre-Rampant P, Hribova E, Bernard M, Lukaszewski A, Dolezel J, Chalhoub B (2006) Advanced resources for plant genomics: a BAC library specific for the short arm of wheat chromosome 1B. Plant J 47:977-986

Jewell DC, Islam-Faridi N (1994) A Technique for Somatic Chromosome Preparation and Cbanding of Maize. In: Freeling M, Walbot V (eds) The Maize Handbook. SpringerVerlag, New York, Inc, pp 484-493

Jiang JM (2019) Fluorescence in situ hybridization in plants: recent developments and future applications. Chromosome Res 27:153-165

Jiang JM, Gill BS (1994) Nonisotopic in situ hybridization and plant genome mapping: the first 10 years. Genome 37:717-725

Jiang JM, Gill BS (2006) Current status and the future of fluorescence in situ hybridization (FISH) in plant genome research. Genome 49:1057-1068

Kakeda K, Yamagata H, Fukui K, Ohno M, Fukui K, Wei ZZ, Zhu FS (1990) High resolution bands in maize chromosomes by G-banding methods. Theor Appl Genet 80:265-272

Kato A (1999) Air drying method using nitrous oxide for chromosome counting in maize. Biotech Histochem 74:160-166

Kato A, Lamb JC, Birchler JA (2004) Chromosome painting using repetitive DNA sequences as probes for somatic chromosome identification in maize. P Natl Acad Sci USA 101:13554-13559 
Kirov I, Khrustaleva L, Laere KV, Soloviev A, Sofie M, Romanov D, Fesenko I (2017) DRAWID: user-friendly java software for chromosome measurements and idiogram drawing. Comp Cytogenet 11:747-757

Koumbaris GL, Bass HW (2003) A new single-locus cytogenetic mapping system for maize (Zea mays L.): overcoming FISH detection limits with marker-selected sorghum $(S$. propinquum L.) BAC clones. Plant J 35:647-659

Lamb JC, Danilova T, Bauer MJ, Meyer JM, Holland JJ, Jensen MD, Birchler JA (2007a) Single-gene detection and karyotyping using small-target fluorescence in situ hybridization on maize somatic chromosomes. Genetics 175:1047-1058

Lamb JC, Meyer JM, Corcoran B, Kato A, Han FP, Birchler JA (2007b) Distinct chromosomal distributions of highly repetitive sequences in maize. Chromosome Res 15:33-49

Lengerova M, Kejnovsky E, Hobza R, Macas J, Grant SR, Vyskot B (2004) Multicolor FISH mapping of the dioecious model plant, Silene latifolia. Theor Appl Genet 108:1193-1199

Levan A, Fredga K, Sandberg AA (1964) Nomenclature for centromeric position on chromosomes. Hereditas-Genetisk A 52:201-220

Li H, Durbin R (2009) Fast and accurate short read alignment with Burrows-Wheeler transform. Bioinformatics 25:1754-1760

Li H, Wang CY, Fu SL, Guo X, Yang BJ, Chen CH, Zhang H, Wang YJ, Liu XL, Han FP, Ji WQ (2014) Development and discrimination of 12 double ditelosomics in tetraploid wheat cultivar DR147. Genome 57:89-95

Liu XY, Sun S, Wu Y, Zhou Y, Gu SW, Yu HX, Yi CD, Gu MH, Jiang JM, Liu B, Zhang T, Gong ZY (2020) Dual-color oligo-FISH can reveal chromosomal variations and evolution in Oryza species. Plant J 101:112-121

Mano Y, Omori F (2013) Flooding tolerance in interspecific introgression lines containing chromosome segments from teosinte (Zea nicaraguensis) in maize (Zea mays subsp mays). Ann Bot-London 112:1125-1139

Martins LD, Yu F, Zhao HN, Dennison T, Lauter N, Wang HY, Deng ZH, Thompson A, Semrau K, Rouillard JM, Birchler JA, Jiang JM (2019) Meiotic crossovers characterized by haplotype-specific chromosome painting in maize. Nat Commun 10:4604

McClintock B (1929) Chromosome morphology in Zea mays. Science 69:629-629

Meng Z, Han J, Lin Y, Zhao Y, Lin Q, Ma X, Wang J, Zhang M, Zhang L, Yang Q, Wang K (2020) Characterization of a Saccharum spontaneum with a basic chromosome number of $\mathrm{x}=10$ provides new insights on genome evolution in genus Saccharum. Theor Appl Genet 133:187-199

Meng Z, Zhang ZL, Yan TY, Lin QF, Wang Y, Huang WY, Huang YJ, Li ZJ, Yu QY, Wang JP, Wang K (2018) Comprehensively characterizing the cytological features of Saccharum spontaneum by the development of a complete set of chromosome-specific oligo probes. Front Plant Sci 9:1624

Mukai Y, Nakahara Y, Yamamoto M (1993) Simultaneous discrimination of the three genomes in hexaploid wheat by multicolor fluorescence insitu hybridization using total genomic and highly repeated DNA probes. Genome 36:489-494

Poggio L, Gonzalez G, Confalonieri V, Comas C, Naranjo CA (2005) The genome organization and diversification of maize and its allied species revisited: evidences from classical and FISH-GISH cytogenetic analysis. Cytogenet Genome Res 109:259-267 
Qu MM, Li KP, Han YL, Chen L, Li ZY, Han YH (2017) Integrated karyotyping of woodland strawberry (Fragaria vesca) with oligopaint FISH probes. Cytogenet Genome Res 153:158-164

Rayburn AL, Price HJ, Smith JD, Gold JR (1985) C-band heterochromatin and DNA content in Zea mays. Am J Bot 72:1610-1617

Ross KJ, Fransz P, Jones GH (1996) A light microscopic atlas of meiosis in Arabidopsis thaliana. Chromosome Res 4:507-516

Ross-Ibarra J, Tenaillon M, Gaut BS (2009) Historical divergence and gene flow in the genus Zea. Genetics 181:1397-1409

Sadder MT, Weber G (2001) Karyotype of maize (Zea mays L.) mitotic metaphase chromosomes as revealed by fluorescence in situ hybridization (FISH) with cytogenetic DNA markers. Plant Mol Biol Rep 19:117-123

Sarkinen T, Bohs L, Olmstead RG, Knapp S (2013) A phylogenetic framework for evolutionary study of the nightshades (Solanaceae): a dated 1000-tip tree. Bmc Evol Biol 13:214

Schnable PS, Ware D, Fulton RS, Stein JC, Wei FS, Pasternak S, Liang CZ, Zhang JW, Fulton L, Graves TA et al. (2009) The B73 maize genome: Complexity, diversity, and dynamics. Science 326:1112-1115

Simonikova D, Nemeckova A, Karafiatova M, Uwimana B, Swennen R, Dolezel J, Hribova E (2019) Chromosome painting facilitates anchoring reference genome sequence to chromosomes in situ and integrated karyotyping in Banana (Musa Spp.). Front Plant Sci 10:1503

Song XY, Song RR, Zhou JW, Yan WK, Zhang T, Sun HJ, Xiao J, Wu YF, Xi ML, Lou QF, Wang HY, Wang X (2020) Development and application of oligonucleotide-based chromosome painting for chromosome 4D of Triticum aestivum L. Chromosome Res:10.1007/s10577-10020-09627-10570

Swigonova Z, Lai JS, Ma JX, Ramakrishna W, Llaca V, Bennetzen JL, Messing J (2004) Close split of sorghum and maize genome progenitors. Genome Res 14:1916-1923

Sybenga J (1972) General Cytogenetics. American Elsevier Publishing Co., INC

Ting YC (1965) Spontaneous chromosome inversions of Guatemalan teosintes (Zea mexicana). Genetica 36:229-242

Wang CJR, Harper L, Cande WZ (2006) High-resolution single-copy gene fluorescence in situ hybridization and its use in the construction of a cytogenetic map of maize chromosome 9. Plant Cell 18:529-544

Wang Y, Diehl A, Wu FN, Vrebalov J, Giovannoni J, Siepel A, Tanksley SD (2008) Sequencing and comparative analysis of a conserved syntenic segment in the solanaceae. Genetics 180:391-408

Ward EJ (1980) Banding patterns in maize mitotic chromosomes. Can J Genet Cytol 22:61-67

Wu FN, Tanksley SD (2010) Chromosomal evolution in the plant family Solanaceae. BMC Genomics 11:182

Xin HY, Zhang T, Han YH, Wu YF, Shi JS, Xi ML, Jiang JM (2018) Chromosome painting and comparative physical mapping of the sex chromosomes in Populus tomentosa and Populus deltoides. Chromosoma 127:313-321

Xin HY, Zhang T, Wu YF, Zhang WL, Zhang PD, Xi ML, Jiang JM (2020) An extraordinarily stable karyotype of the woody Populus species revealed by chromosome painting. Plant $\mathrm{J}$ $101: 253-264$ 
Xiong ZY, Pires JC (2011) Karyotype and identification of all homoeologous chromosomes of allopolyploid Brassica napus and its diploid progenitors. Genetics 187:37-49

Zhang P, Li WL, Fellers J, Friebe B, Gill BS (2004) BAC-FISH in wheat identifies chromosome landmarks consisting of different types of transposable elements. Chromosoma 112:288299

Zhao HN, Zhu XB, Wang K, Gent JI, Zhang WL, Dawe RK, Jiang JM (2016) Gene expression and chromatin modifications associated with maize centromeres. G3-Genes Genom Genet 6:183-192 


\section{Figure legends}

Figure 1. Development of barcode oligo-FISH probes for maize chromosome identification. (a) Predicted locations of the oligo-FISH signals on 10 maize chromosomes. Oligos were selected from a total of 24 chromosomal regions (12 red regions and 12 green regions). The 10 chromosomes can be distinguished from each other based on number and location of the $\mathrm{red} /$ green signals. The centromere positions on the 10 chromosomes in the maize reference genome were based on the locations of sequences associated with CENH3 nucleosomes (Zhao et al., 2016). (b) FISH mapping of the two oligo-FISH probes on metaphase chromosomes prepared from maize inbred B73. Bar $=10 \mu \mathrm{m}$. (c) Homologous chromosomes were digitally excised from (b) and paired. The centromeres of the chromosomes are aligned by a dotted line.

Figure 2. FISH mapping of the two oligo-FISH probes on metaphase chromosomes prepared from (a) Z. mays ssp. parviglumis; (b) Z. mays ssp. mexicana; (c) Z. mays ssp. huehuetenangensis; (d) Z. diploperennis; (e) Z. nicaraguensis; and (f) Z. luxurians. Bars = 10 $\mu \mathrm{m}$.

Figure 3. Comparative karyotyping of seven Zea species and subspecies. Chromosomes 1-10 from each species/subspecies are arranged from left to right. The karyotypes were developed from the same metaphase cells in Figure $1 \mathrm{~b}$ for maize and Figure 2 for other species/subspecies. Double arrows point to the two green signals on the long arm of chromosome 4 of $Z$. nicaraguensis and Z. luxurians. Both signals are located in the interstitial regions. Yellow arrowheads point to a knob on chromosome 5, which is located in the subterminal region in $Z$. mays ssp. parviglumis, but at the distal end in Z. nicaraguensis and Z. luxurians. Red arrowheads point to knobs observed only on one of the two homologous chromosomes. White arrowheads point to knobs that show visibly different sizes on homologous chromosomes.

Figure 4. Chromosome identification in a tetraploid species $Z$. perennis. The top panel shows a complete metaphase cell hybridized with the two oligo-FISH probes. The bottom panel shows the 4 homologous chromosomes of each of the 10 Z. perennis chromosomes digitally excised from the same cell. $\mathrm{Bar}=10 \mu \mathrm{m}$. 
bioRxiv preprint doi: https://doi.org/10.1101/2020.01.22.915942; this version posted March 18, 2020. The copyright holder for this preprint (which was not certified by peer review) is the author/funder. All rights reserved. No reuse allowed without permission.

Figure 5. FISH mapping of the two oligo-FISH probes on metaphase chromosomes prepared from (a) Tripsacum dactyloides $(2 \mathrm{n}=2 \mathrm{x}=36)$; and (b) Sorghum bicolor $(2 \mathrm{n}=2 \mathrm{x}=20)$. Bars $=$ $10 \mu \mathrm{m}$. 
Table 1. Relative length of individual chromosomes of maize and its wild relatives.

\begin{tabular}{c|cccccc}
\hline Chr. & Z. mays & $\begin{array}{c}\text { Z. mays ssp. } \\
\text { parviglumis }\end{array}$ & $\begin{array}{c}\text { Z. mays ssp. } \\
\text { mexicana }\end{array}$ & $\begin{array}{c}\text { Z. mays ssp. } \\
\text { huehuetenangensis }\end{array}$ & Z. nicaraguensis & Z. luxurians \\
\hline 1 & $14.26 \pm 1.22$ & $12.56 \pm 0.41$ & $13.26 \pm 0.50$ & $13.66 \pm 0.50$ & $13.16 \pm 0.62$ & $13.41 \pm 0.76$ \\
2 & $11.19 \pm 0.28$ & $11.56 \pm 0.30$ & $12.89 \pm 0.68$ & $12.11 \pm 0.72$ & $12.26 \pm 0.94$ & $11.75 \pm 0.61$ \\
3 & $10.76 \pm 0.54$ & $11.49 \pm 0.38$ & $10.23 \pm 0.49$ & $11.22 \pm 0.32$ & $10.66 \pm 0.46$ & $10.41 \pm 0.45$ \\
4 & $11.28 \pm 0.53$ & $12.10 \pm 0.39$ & $12.12 \pm 0.41$ & $11.04 \pm 0.55$ & $11.14 \pm 0.52$ & $11.14 \pm 0.63$ \\
5 & $10.36 \pm 0.22$ & $10.67 \pm 0.36$ & $10.34 \pm 0.55$ & $10.65 \pm 0.43$ & $11.46 \pm 0.42$ & $12.25 \pm 0.56$ \\
6 & $8.51 \pm 0.34$ & $9.04 \pm 0.42$ & $8.06 \pm 0.48$ & $10.03 \pm 0.18$ & $8.10 \pm 0.57$ & $8.58 \pm 0.74$ \\
7 & $9.44 \pm 0.50$ & $8.90 \pm 0.42$ & $9.40 \pm 0.46$ & $10.44 \pm 1.13$ & $9.21 \pm 0.27$ & $8.49 \pm 0.32$ \\
8 & $9.18 \pm 0.33$ & $8.87 \pm 0.37$ & $8.79 \pm 0.42$ & $10.26 \pm 1.05$ & $8.69 \pm 0.42$ & $7.89 \pm 0.50$ \\
9 & $8.03 \pm 0.36$ & $8.56 \pm 0.61$ & $8.00 \pm 0.31$ & $9.94 \pm 0.96$ & $7.78 \pm 0.41$ & $8.19 \pm 0.40$ \\
10 & $6.99 \pm 0.46$ & $6.29 \pm 0.33$ & $6.91 \pm 0.37$ & $8.49 \pm 1.28$ & $7.53 \pm 0.66$ & $7.90 \pm 0.30$ \\
\hline
\end{tabular}

Each chromosomal arm was measured in 10 metaphase cells of maize and its wild relatives, except for $Z$. mays ssp. parviglumis with only 7 metaphase cells. 
Table 2. Arm ratio of individual chromosomes of maize and its wild relatives.

\begin{tabular}{c|cccccc}
\hline Chr. & Z. mays & $\begin{array}{c}\text { Z. mays ssp. } \\
\text { parviglumis }\end{array}$ & $\begin{array}{c}\boldsymbol{Z} \text {. mays ssp. } \\
\text { mexicana }\end{array}$ & $\begin{array}{c}\boldsymbol{Z} \text {. mays ssp. } \\
\text { huehuetenangensis }\end{array}$ & $\begin{array}{c}\text { Z. nicaraguensis } \\
\text { Z. luxurians }\end{array}$ \\
\hline 1 & $1.26 \pm 0.14 \mathrm{~m}$ & $1.22 \pm 0.16 \mathrm{~m}$ & $1.25 \pm 0.11 \mathrm{~m}$ & $1.26 \pm 0.27 \mathrm{~m}$ & $1.11 \pm 0.12 \mathrm{~m}$ & $1.16 \pm 0.12 \mathrm{~m}$ \\
2 & $1.52 \pm 0.18 \mathrm{~m}$ & $1.49 \pm 0.36 \mathrm{~m}$ & $1.83 \pm 0.32 \mathrm{sm}$ & $1.43 \pm 0.21 \mathrm{~m}$ & $1.38 \pm 0.23 \mathrm{~m}$ & $1.27 \pm 0.17 \mathrm{~m}$ \\
3 & $1.65 \pm 0.28 \mathrm{~m}$ & $1.61 \pm 0.27 \mathrm{~m}$ & $1.89 \pm 0.34 \mathrm{sm}$ & $1.53 \pm 0.19 \mathrm{~m}$ & $1.44 \pm 0.18 \mathrm{~m}$ & $1.59 \pm 0.19 \mathrm{~m}$ \\
4 & $1.34 \pm 0.17 \mathrm{~m}$ & $1.51 \pm 0.32 \mathrm{~m}$ & $1.60 \pm 0.30 \mathrm{~m}$ & $1.26 \pm 0.17 \mathrm{~m}$ & $1.55 \pm 0.21 \mathrm{~m}$ & $1.23 \pm 0.17 \mathrm{~m}$ \\
5 & $1.54 \pm 0.45 \mathrm{~m}$ & $1.59 \pm 0.25 \mathrm{~m}$ & $1.68 \pm 0.32 \mathrm{~m}$ & $1.31 \pm 0.35 \mathrm{~m}$ & $1.34 \pm 0.27 \mathrm{~m}$ & $1.16 \pm 0.16 \mathrm{~m}$ \\
6 & $2.40 \pm 0.46 \mathrm{sm}$ & $3.31 \pm 0.90 \mathrm{a}$ & $2.74 \pm 0.63 \mathrm{sm}$ & $2.39 \pm 0.39 \mathrm{sm}$ & $3.32 \pm 1.06 \mathrm{a}$ & $3.56 \pm 0.60 \mathrm{a}$ \\
7 & $2.11 \pm 0.46 \mathrm{sm}$ & $2.53 \pm 0.58 \mathrm{sm}$ & $1.51 \pm 0.37 \mathrm{~m}$ & $1.86 \pm 0.22 \mathrm{sm}$ & $2.58 \pm 0.65 \mathrm{sm}$ & $2.39 \pm 0.35 \mathrm{sm}$ \\
8 & $2.13 \pm 0.48 \mathrm{sm}$ & $2.78 \pm 0.75 \mathrm{sm}$ & $1.97 \pm 0.47 \mathrm{sm}$ & $1.69 \pm 0.33 \mathrm{~m}$ & $1.99 \pm 0.48 \mathrm{sm}$ & $2.29 \pm 0.32 \mathrm{sm}$ \\
9 & $1.80 \pm 0.34 \mathrm{sm}$ & $1.58 \pm 0.29 \mathrm{~m}$ & $1.88 \pm 0.35 \mathrm{sm}$ & $1.41 \pm 0.34 \mathrm{~m}$ & $2.77 \pm 0.58 \mathrm{sm}$ & $2.89 \pm 0.63 \mathrm{sm}$ \\
10 & $1.78 \pm 0.40 \mathrm{sm}$ & $1.99 \pm 0.20 \mathrm{sm}$ & $1.83 \pm 0.37 \mathrm{sm}$ & $1.99 \pm 0.36 \mathrm{sm}$ & $2.53 \pm 0.38 \mathrm{sm}$ & $2.81 \pm 0.39 \mathrm{sm}$ \\
\hline
\end{tabular}

Each chromosomal arm was measured in 10 metaphase cells of maize and its wild relatives, except for $Z$. mays ssp. parviglumis with only 7 metaphase cells. $\mathrm{m}=$ metacentric, $\mathrm{sm}=$ submetacentric, $\mathrm{a}=$ acrocentric. 


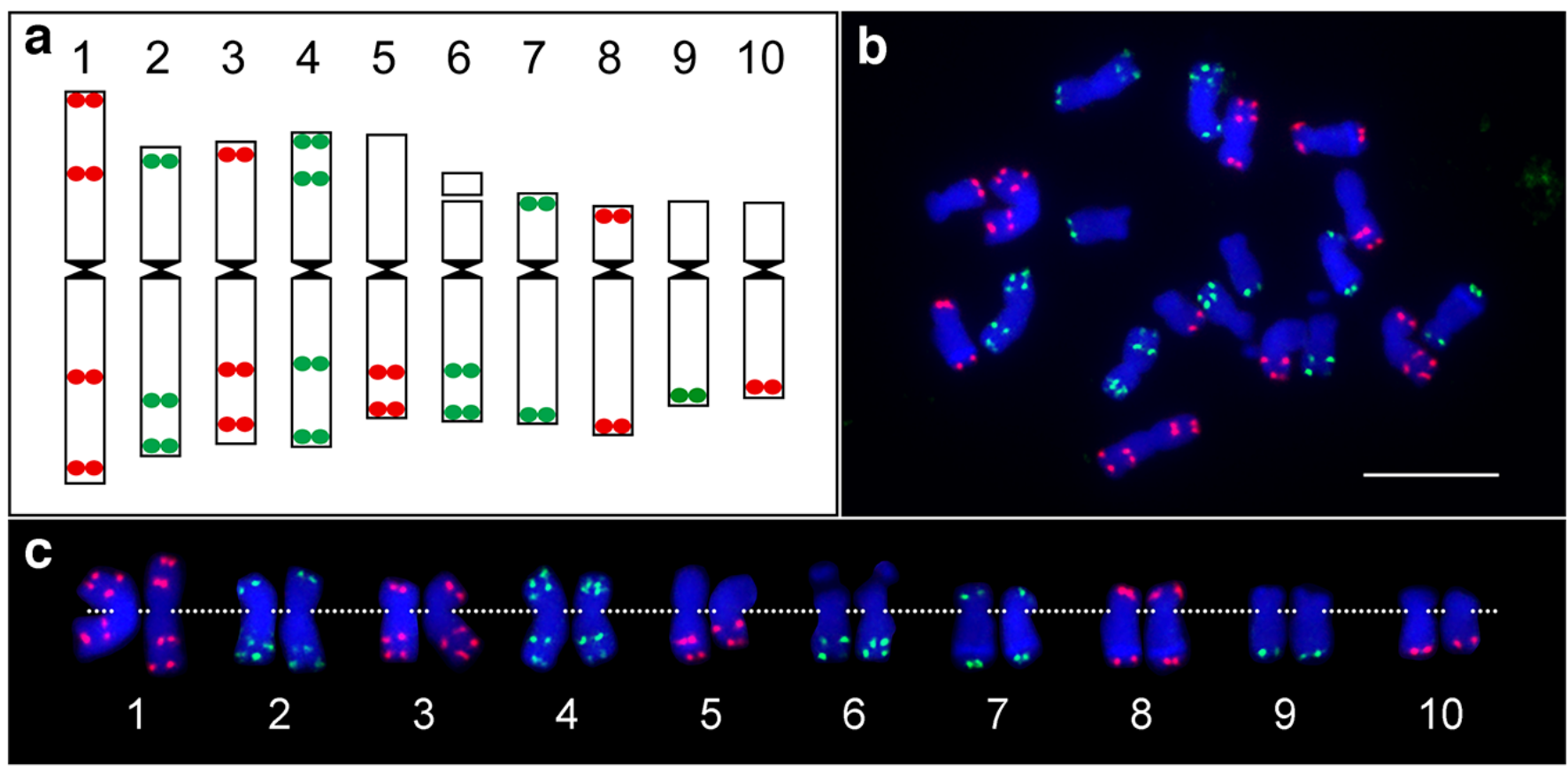

Figure 1. Development of barcode oligo-FISH probes for maize chromosome identification. (a) Predicted locations of the oligoFISH signals on 10 maize chromosomes. Oligos were selected from a total of 24 chromosomal regions (12 red regions and 12 green regions). The 10 chromosomes can be distinguished from each other based on number and location of the red/green signals. The centromere positions on the 10 chromosomes in the maize reference genome were based on the locations of sequences associated with CENH3 nucleosomes (Zhao et al., 2016). (b) FISH mapping of the two oligo-FISH probes on metaphase chromosomes prepared from maize inbred B73. Bar $=10 \mu \mathrm{m}$. (c) Homologous chromosomes were digitally excised from (b) and paired. The centromeres of the chromosomes are aligned by a dotted line. 


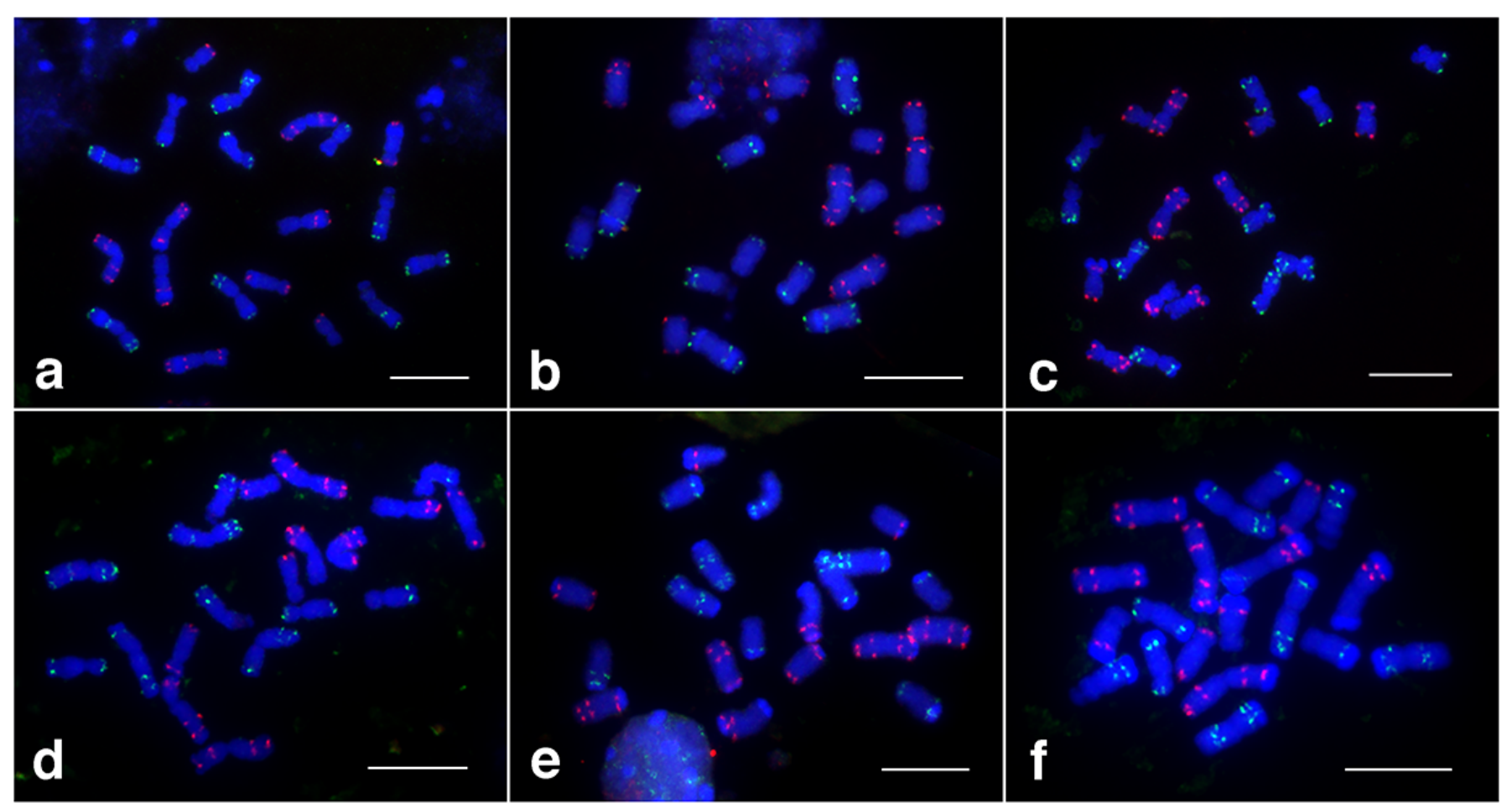

Figure 2. FISH mapping of the two oligo-FISH probes on metaphase chromosomes prepared from (a) $Z$. mays ssp. parviglumis; (b) Z. mays ssp. mexicana; (c) Z. mays ssp. huehuetenangensis; (d) Z. diploperennis; (e) Z. nicaraguensis; and (f) $Z$. luxurians. Bars $=10 \mu \mathrm{m}$. 


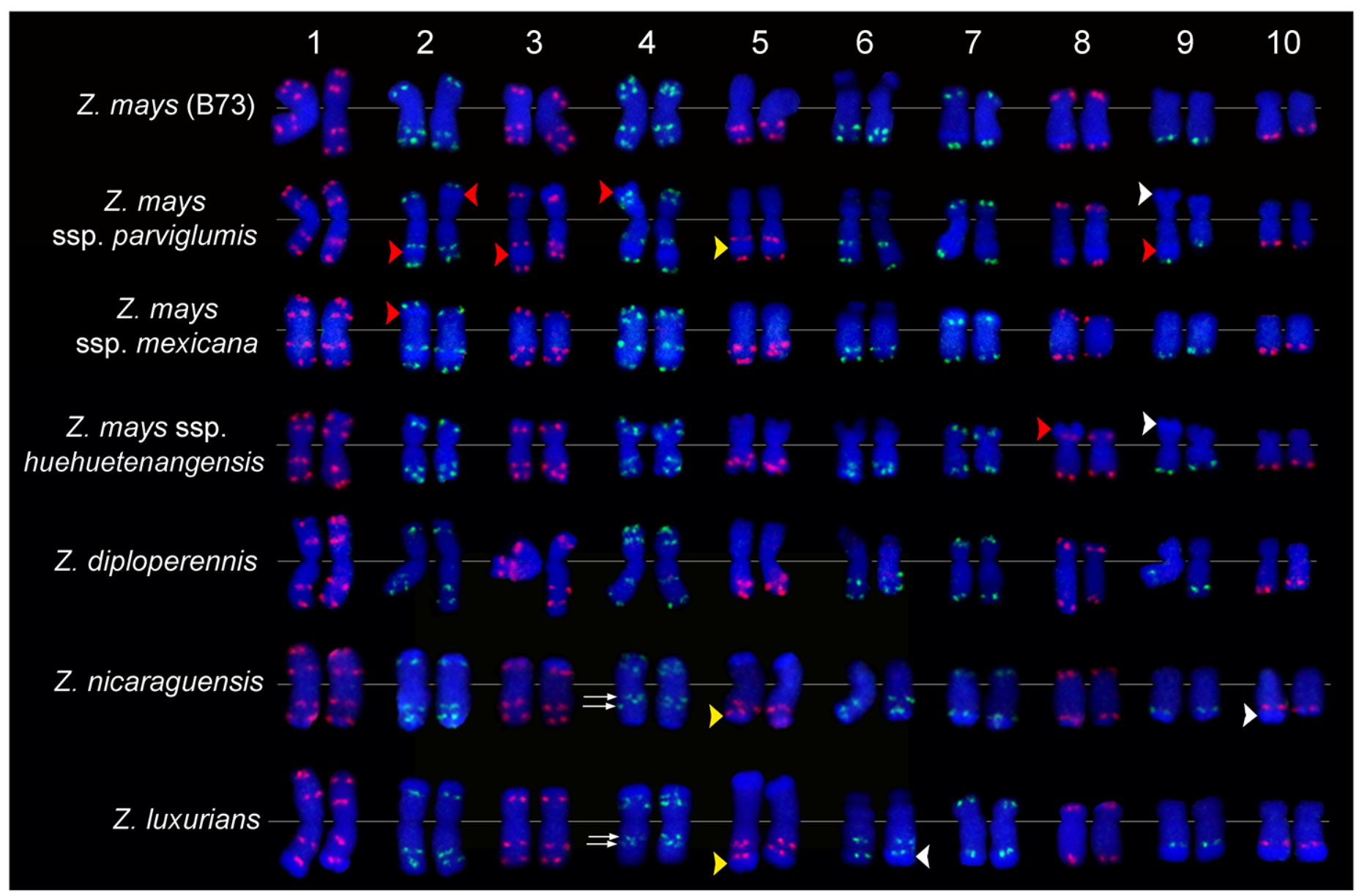

Figure 3. Comparative karyotyping of seven Zea species and subspecies. Chromosomes 1-10 from each species/subspecies are arranged from left to right. The karyotypes were developed from the same metaphase cells in Figure $1 \mathrm{~b}$ for maize and Figure 2 for other species/subspecies. Double arrows point to the two green signals on the long arm of chromosome 4 of $Z$. nicaraguensis and Z. luxurians. Both signals are located in the interstitial regions. Yellow arrowheads point to a knob on chromosome 5, which is located in the subterminal region in Z. mays ssp. parviglumis, but at the distal end in Z. nicaraguensis and Z. luxurians. Red arrowheads point to knobs observed only on one of the two homologous chromosomes. White arrowheads point to knobs that show visibly different sizes on homologous chromosomes. 


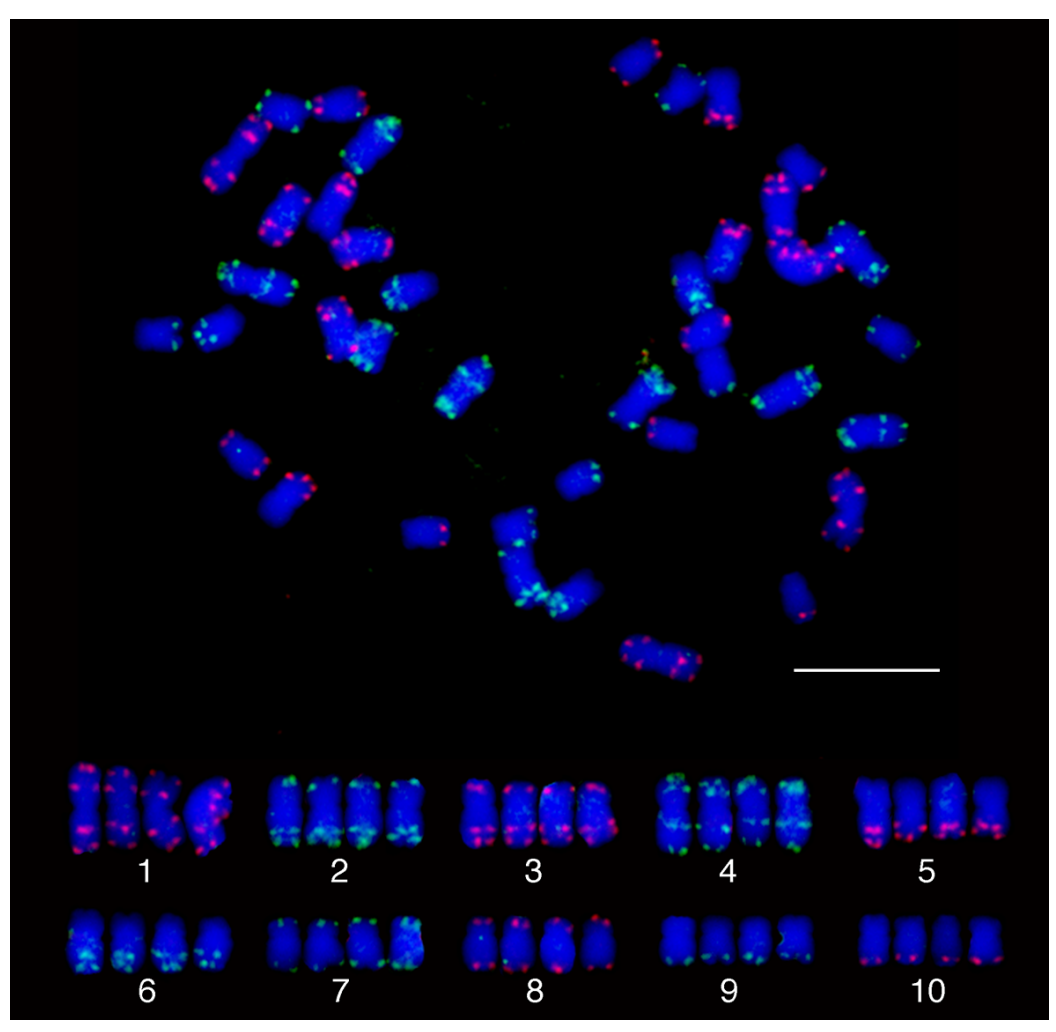

Figure 4. Chromosome identification in a tetraploid species $Z$. perennis. The top panel shows a complete metaphase cell hybridized with the two oligo-FISH probes. The bottom panel shows the 4 homologous chromosomes of each of the 10 Z. perennis chromosomes digitally excised from the same cell. Bar $=10 \mu \mathrm{m}$. 


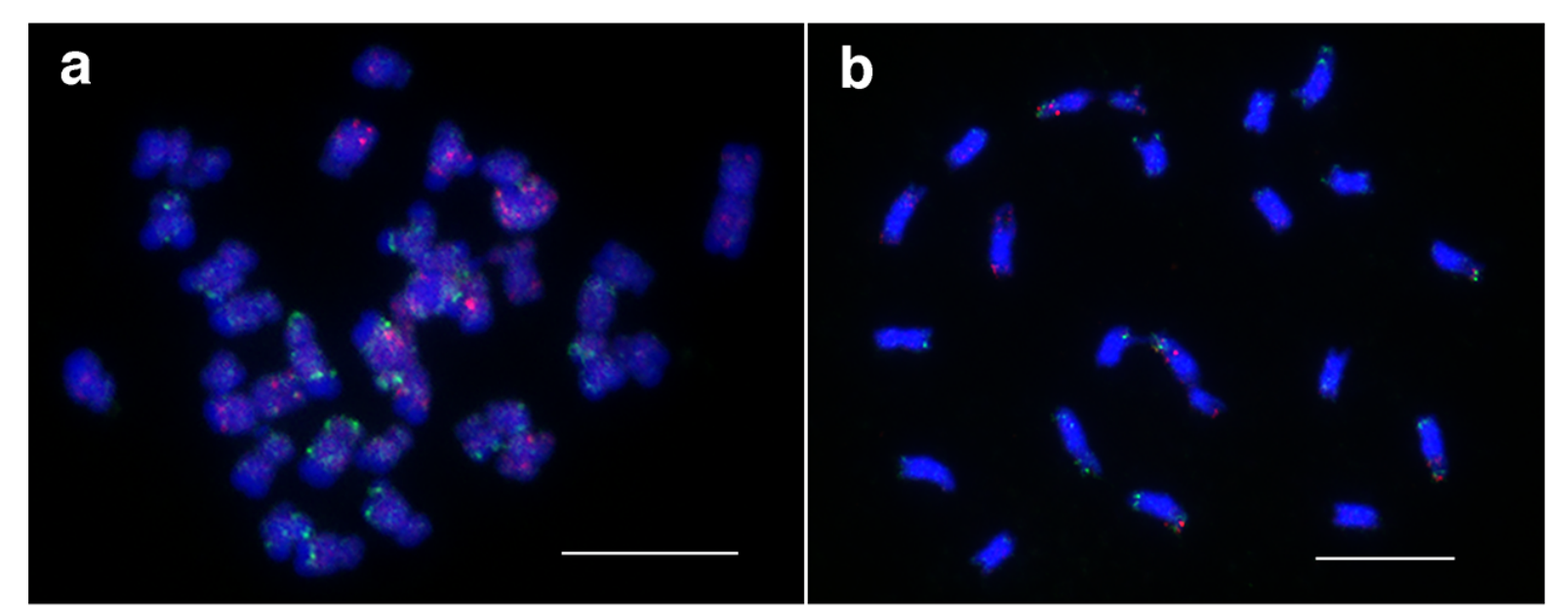

Figure 5. FISH mapping of the two oligo-FISH probes on metaphase chromosomes prepared from (a) Tripsacum dactyloides $(2 \mathrm{n}=2 \mathrm{x}=36)$; and (b) Sorghum bicolor $(2 \mathrm{n}=2 \mathrm{x}=20)$. Bars $=10 \mu \mathrm{m}$. 